\title{
Estudo epidemiológico da hanseníase no Brasil: reflexão sobre as metas de eliminação
}

\author{
Mara Dayanne Alves Ribeiro, ${ }^{1}$ Jefferson Carlos Araujo Silva ${ }^{2}$ e \\ Sabrynna Brito Oliveira ${ }^{3}$
}

Como citar Ribeiro MDA, Silva JCA, Oliveira SB. Estudo epidemiológico da hanseníase no Brasil: reflexão sobre as metas de eliminação. Rev Panam Salud Publica. 2018;42:e42. https:/ / doi.org/10.26633/RPSP.2018.42

RESUMO Objetivos. Descrever o perfil epidemiológico da hanseníase no Brasil no período de 2005 a 2015 e verificar como os indicadores brasileiros estão se comportando em relação às metas estipuladas pela Organização Mundial da Saúde (OMS) para eliminação dessa doença.

Métodos. A pesquisa foi realizada a partir dos dados disponíveis nos sites do Ministério da Saúde. Foram avaliados os indicadores: dados de prevalência, coeficientes de detecção geral e conforme grupo etário (< 15 anos ou $\geq 15$ anos), porcentagem de cura e grau 2 de incapacidade.

Resultados. No período do estudo, o coeficiente de prevalência dos casos de hanseníase manteve-se em patamar médio (de 1,00 a 4,99/10 000 habitantes), com tendência nacional decrescente. Entretanto, esse comportamento não foi observado nas regiões Nordeste, Norte e Centro-Oeste. O coeficiente de casos novos em indivíduos com menos de 15 anos apresentou diminuição entre 2005 e 2015 em todas as regiões. O coeficiente de detecção de casos novos diagnosticados com grau 2 de incapacidade teve redução significativa, principalmente nas regiões Sul e Sudeste; o Norte do país foi a única região com aumento desse coeficiente. O percentual de cura de hanseníase no Brasil, independentemente da faixa etária, não sofreu alteração desde 2005, sendo considerado regular (75\% a 90\%).

Conclusão. Os principais indicadores de hanseníase apresentaram redução no período do estudo. Embora o Brasil não tenha erradicado a hanseníase, essa meta deverá ser alcançada em 2020 caso sejam mantidos os parâmetros. Recomenda-se adaptar a política de atenção à hanseníase à realidade de cada região brasileira, visto que a prevalência da doença apresenta distribuição heterogênea.

Palavras-chave Epidemiologia; hanseníase; erradicação de doenças; Brasil.

\footnotetext{
Universidade Federal do Ceará (UFC), Campus de Sobral, Sobral (CE), Brasil. Correspondência: mara_dayanne2@hotmail.com

2 Hospital Universitário da Universidade Federal do Maranhão (HU-UFMA), Unidade Presidente Dutra, São Luís (MA), Brasil.

3 Universidade Federal de Minas Gerais (UFMG), Instituto de Ciências Biológicas, Departamento de Microbiologia, Belo Horizonte (MG), Brasil.
}

A hanseníase representa um problema de saúde pública pelo seu poder de causar incapacidade física, social e econômica. Em 1991, após adoção da poliquimioterapia como tratamento específico, a Organização Mundial da Saúde (OMS) propôs a eliminação da hanseníase como problema de saúde pública até o ano 2000, sendo a eliminação definida como prevalência conhecida inferior a 1/10 000 habitantes. Desse modo, a OMS almeja que a prevalência da doença não influencie a produtividade social nem o desenvolvimento das comunidades. 
As metas de eliminação são, proporcionalmente, mais viáveis quanto maior a capilaridade da atenção primária à saúde (APS) e quanto melhor a qualidade do serviço de saúde (1).

Nesse contexto, o Brasil, assim como os outros países, implementou políticas de tratamento ambulatorial, campanhas e diretrizes para controle da doença. Por intermédio do Plano Nacional de Eliminação da Hanseníase, os estados e municípios promoveram ações como oficialização da implantação da poliquimioterapia e diagnóstico e tratamento para todos os casos novos esperados. Além disso, recebeu ênfase o diagnóstico precoce (objetivo de diagnosticar 90\% dos casos novos antes do aparecimento de deformidades físicas), a promoção de alta por cura em $80 \%$ dos casos que iniciaram o tratamento e a redução da taxa de prevalência em $15 \%$ a $20 \%$ ao ano (2). Ademais, no ano 2000, o Ministério da Saúde iniciou a publicação de diretrizes destinadas a orientar as medidas de prevenção, diagnóstico, tratamento e controle de hanseníase em todos os níveis do Sistema Único de Saúde (SUS), principalmente na APS, para viabilizar a descentralização da assistência à doença. Além das diretrizes, a criação de legislação específica facilitou a implementação do diagnóstico e do tratamento precoces em unidades básicas de saúde (3).

No começo de 2005, a eliminação da hanseníase havia sido alcançada em todos os países exceto nove: Angola, Brasil, Índia, Madagascar, Moçambique, Nepal, República Centro-Africana, República Democrática do Congo e Tanzânia (4). O Brasil não alcançou a meta mundial, mas concordou em se empenhar no controle da doença usando o impacto político da campanha global pela eliminação e um prazo estendido até o ano de 2010. Entretanto, apesar dos esforços de todas as esferas do governo brasileiro, o objetivo não foi alcançado, e segue com novo prazo até $2020(5,6)$.

Após o estabelecimento da segunda meta de eliminação em 2005, consequente não alcance em 2010 e instituição de uma nova meta para 2020, observou-se uma diminuição nos coeficientes de prevalência da hanseníase em alguns países endêmicos (6). Assim, o objetivo do presente estudo foi descrever o comportamento epidemiológico da hanseníase no Brasil no período de 2005 a 2015 a fim de observar como os indicadores brasileiros estão se comportando em relação às metas estipuladas pela OMS.

\section{MATERIAIS E MÉTODOS}

Este é um estudo transversal, descritivo, retrospectivo e quantitativo, realizado no período de julho de 2016 a junho de 2017. Os dados foram coletados a partir das informações disponíveis nos sites TABNET/DATASUS (http://www2. datasus.gov.br/DATASUS / index. php?area=02) e Portal da Saúde (http:/ / portalms.saude.gov.br/) do Ministério da Saúde. No Portal, os dados foram obtidos seguindo os passos: O ministério > Secretaria de vigilância em saúde $>$ Vigilância de A a Z > Hanseníase > Situação epidemiológica/dados (http:/ / portalms. saude.gov.br/saude-de-a-z/hanseniase/ situacao-epidemiologica).

Delimitou-se a amostra ao período de 2005 a 2015, correspondente ao período entre as duas últimas metas de eliminação da doença até o presente momento. Os dados oficiais de 2016 e 2017 ainda não foram disponibilizados pelo Ministério da Saúde.

Para classificação da endemicidade da hanseníase, são utilizados coeficientes de prevalência e de detecção geral, além de coeficiente de detecção em menores de 15 anos, porcentagem de cura e coeficiente de detecção de casos novos diagnosticados com grau 2 de incapacidade (2). Assim, foram esses foram os indicadores considerados para análise.

O grau de incapacidade é determinado a partir de avaliação neurológica dos olhos, mãos e pés e pode ser classificado em 0,1 e 2, sendo esse último o mais agressivo. Por sua vez, o coeficiente de prevalência é classificado como baixo (0,00 a 0,99/10 000 habitantes), médio (de 1,00 a 4,99/10 000 habitantes), alto (5,00 a 9,99/10 000 habitantes), muito alto $(10,00$ a 19,99/10 000 habitantes) e hiperendêmico (acima de 20,00/10 000 habitantes). O percentual de cura foi denominado bom quando $>90 \%$, regular para a faixa de 75 a $90 \%$ e precário quando $<75 \%$ (7).

A análise foi realizada através de estatística descritiva simples e comparativa entre as diferentes regiões brasileiras e entre os períodos de 2005 a 2009 e 2010 a 2015 , compreendidos como marcos relacionados às metas estabelecidas pela OMS a partir de 2000.

\section{RESULTADOS}

A prevalência de casos de hanseníase no Brasil no ano de 2015 foi de 1,01/10 000 habitantes - a menor taxa registrada nos últimos 11 anos (figura 1). Durante esse período, o coeficiente de prevalência oscilou entre 1,01 e 2,11/10 000 habitantes. Os coeficientes mais elevados foram registrados entre os anos de 2005 e 2009. Desde 2005, o coeficiente de prevalência do país permaneceu classificado como médio (de 1,00 a 4,99/10 000 habitantes) (7).

Apesar da tendência nacional de diminuição da prevalência, esse comportamento não foi observado em todas as regiões brasileiras. As regiões Norte,

\section{FIGURA 1. Coeficiente de prevalência de hanseníase no Brasil, 2005 a $2015^{a}$}

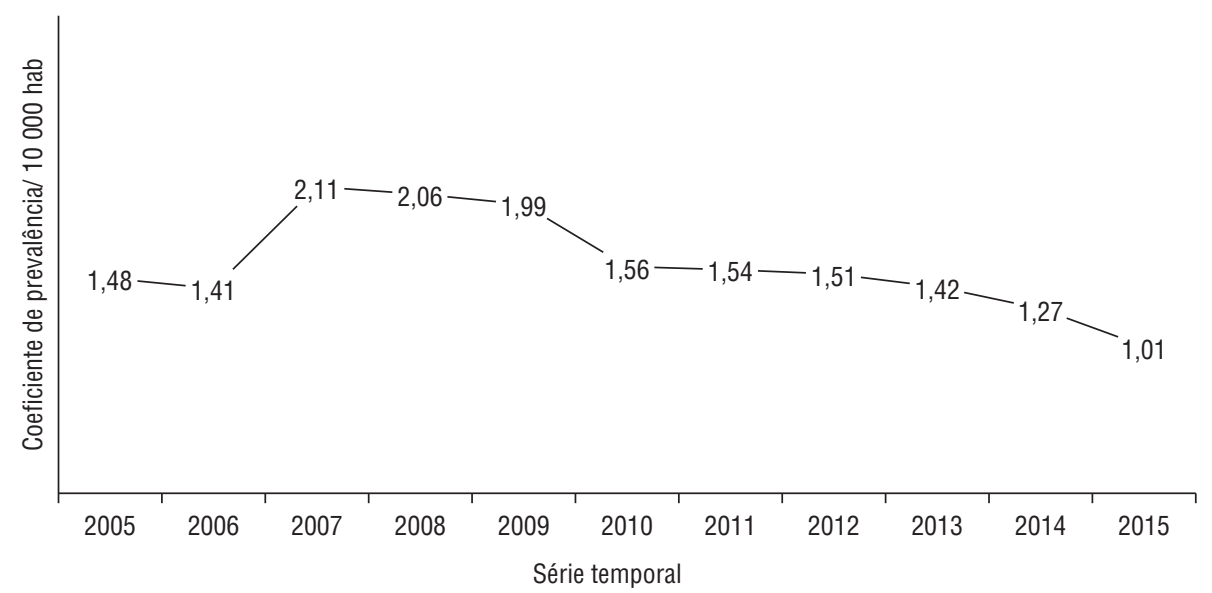

a Classificação do coeficiente de prevalência no Brasil: baixo, 0,00 a 0,99/10 000 habitantes; médio, 1,00 a 4,99/10 000 habitantes; alto, 5,00 a 9,99/10 000 habitantes; muito alto, 10,00 a 19,99/10 000 habitantes; e hiperendêmico, acima de 20,00/10 000 habitantes (7). 
Nordeste e Centro-Oeste apresentaram coeficiente de prevalência acima da média nacional no ano de 2015 e explicam a carga endêmica do país (figura 2). Os estados responsáveis pelos altos índices de prevalência nessas regiões no ano de 2015 foram Mato

FIGURA 2. Coeficiente de prevalência da hanseníase nas regiões brasileiras, 2005, 2010 e 2015

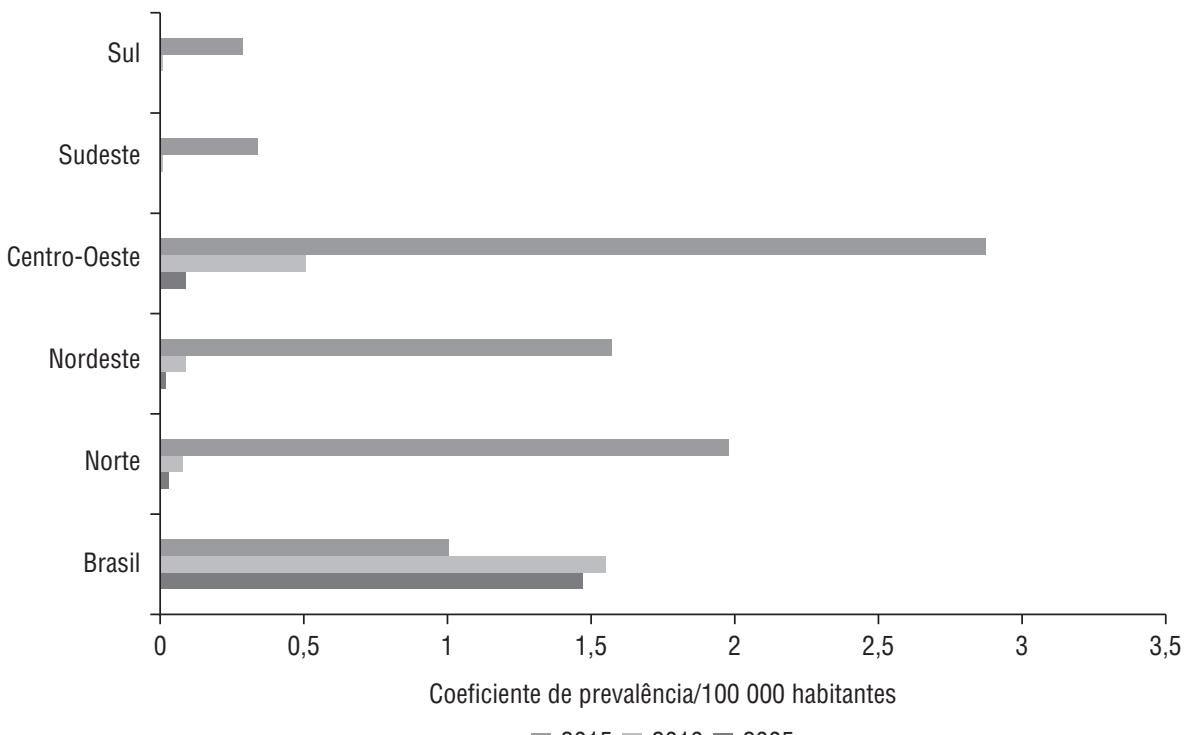

Grosso, no Centro-Oeste (capital "O") (7,75/10 000 habitantes); Tocantins, no Norte (4,2/10 000 habitantes); e Maranhão, no Nordeste (3,76/10 000 habitantes). As regiões Sudeste e Sul não tiveram registro de casos de hanseníase entre 2005 e 2008. Nos demais anos, o

coeficiente de prevalência ficou abaixo da média nacional nessas regiões.

O coeficiente de detecção geral da hanseníase em 2005 foi de 26,86 casos/ 100000 habitantes, sendo considerado muito alto $(20,00$ a 39,99/100 000 habitantes). Essa classificação permaneceu até o ano de 2009, quando o país registrou coeficiente de detecção geral de 19,64/100 000 habitantes, uma redução de 26,88\% em 5 anos. A partir de 2009, até o ano de 2015, o coeficiente de detecção passou para a categoria "alto", de 10,00 a 19,99/100 000 habitantes.

O coeficiente de casos novos de hanseníase em indivíduos com menos 15 anos, assim como o número absoluto de indivíduos com o primeiro diagnóstico da doença nessa faixa etária, apresentaram diminuição entre 2005 e 2015 para todas as regiões do país (tabela 1). Mesmo com essa redução, o Brasil segue na categoria "médio" para essa faixa etária (coeficiente de detecção de 0,50 a 2,49/100 000 habitantes). A análise por região aponta que o coeficiente de detecção das regiões Norte, Nordeste e Centro-Oeste foi médio (0,50 a 2,49/100 000 habitantes). Por sua vez, o coeficiente das regiões Sudeste e Sul foi classificado como baixo $(<0,50 / 100000$ habitantes).

TABELA 1. Número de casos novos de hanseníase por região do Brasil conforme grupo etário, 2005,2010 e 2015

\begin{tabular}{|c|c|c|c|c|c|c|c|c|}
\hline & \multicolumn{4}{|c|}{$<15$ anos } & \multicolumn{4}{|c|}{$\geq 15$ anos } \\
\hline & 2005 & 2010 & 2015 & Variação ${ }^{\mathrm{a}}(\%)$ & 2005 & 2010 & 2015 & Variação $(\%)$ \\
\hline \multicolumn{9}{|l|}{ Brasil } \\
\hline Número de casos & 3909 & 2509 & 2113 & $-45,97$ & 44842 & 35849 & 28761 & $-35,86$ \\
\hline Coeficiente de detecçãob & 21,22 & 13,15 & 10,33 & $-51,32$ & 26,49 & 18,8 & 14,07 & $-46,88$ \\
\hline \multicolumn{9}{|l|}{ Região Norte } \\
\hline Número de casos & 982 & 632 & 525 & $-46,54$ & 8612 & 6936 & 5147 & $-40,23$ \\
\hline Coeficiente de detecçãob & 6,68 & 3,98 & 2,99 & $-55,24$ & 65,37 & 43,72 & 29,4 & $-55,02$ \\
\hline \multicolumn{9}{|l|}{ Região Nordeste } \\
\hline Número de casos & 1843 & 1235 & 1109 & $-39,83$ & 17718 & 14991 & 12760 & $-27,98$ \\
\hline Coeficiente de detecçãob & 3,61 & 2,32 & 1,96 & $-47,70$ & 38,38 & 28,24 & 22,56 & $-41,22$ \\
\hline \multicolumn{9}{|l|}{ Região Centro-Oeste } \\
\hline Número de casos & 451 & 282 & 296 & $-34,37$ & 7211 & 5942 & 5623 & $-22,02$ \\
\hline Coeficiente de detecção ${ }^{b}$ & 3,46 & 2,0 & 1,91 & $-47,80$ & 58,87 & 42,29 & 36,41 & $-38,15$ \\
\hline \multicolumn{9}{|l|}{ Região Sudeste } \\
\hline Número de casos & 580 & 232 & 154 & $-73,45$ & 9255 & 6252 & 4013 & $-56,64$ \\
\hline Coeficiente de detecçãob & 0,73 & 0,28 & 0,17 & $-76,71$ & 12,53 & 7,78 & 4,86 & $-61,21$ \\
\hline \multicolumn{9}{|l|}{ Região Sul } \\
\hline Número de casos & 51 & 24 & 12 & $-76,47$ & 2015 & 1419 & 1013 & $-49,73$ \\
\hline Coeficiente de detecção $0^{b}$ & 0,18 & 0,08 & 0,07 & $-61,12$ & 7,66 & 5,18 & 3,47 & $-54,70$ \\
\hline \multicolumn{9}{|l|}{ Ignorado/exterior } \\
\hline Número de casos & 2 & 13 & 17 & +750 & 31 & 309 & 205 & $+561,29$ \\
\hline Coeficiente de detecçãob & Não se aplica & & & & & & & \\
\hline
\end{tabular}

a Comparação entre os anos de 2005 e 2015.

b Por 100000 habitantes. 
O número absoluto de casos e o coeficiente de detecção de casos novos em pessoas com idade $\geq 15$ anos também caíram, tanto para o Brasil quanto para todas as regiões. Assim, o coeficiente de detecção passou de hiperendêmico (> 40,00/100 000 habitantes) para muito alto (20,00 a 39,99/100 000 habitantes). Os dados do Sistema de Informação sobre Agravos de Notificação (SINAN) apontam ainda um aumento importante do número de casos com informações preenchidas como "ignoradas" na ficha de notificação compulsória, assim como de casos de brasileiros infectados no país, mas diagnosticados no exterior entre 2005 e 2015 em todas as faixas etárias. O número de casos ignorados/diagnosticados no exterior cresceu de 31 em 2005 para 294 em 2015.

Sabe-se que os principais problemas decorrentes da hanseníase estão relacionados com o grau de incapacidade física dos pacientes. O Ministério da Saúde considera o coeficiente de detecção de casos novos diagnosticados com grau 2 de incapacidade como um parâmetro importante a ser analisado. Entre os anos de em $34,75 \%$, atingindo o menor coeficiente dos últimos 11 anos (0,92/100 000 habitantes). As regiões Sudeste e Sul apresentaram o melhor desempenho entre as regiões, com redução de 55,20\% e $59,74 \%$ do coeficiente de detecção de casos novos, respectivamente. As regiões Nordeste e Centro-Oeste tiveram decréscimo discreto de seus coeficientes $(21,96 \%$ e $13,98 \%$, respectivamente). 2005 a 2015, o Brasil reduziu o coeficiente

A região Norte se destacou como a única que apresentou aumento do coeficiente, de 1,76 em 2005 para 1,83/100 000 habitantes em 2015 (+3,98\%).

Durante o período de análise, o Brasil apresentou um percentual de cura regular, independentemente da faixa etária do paciente (figura 3). De 2005 e 2010, as regiões Sudeste e Sul apresentaram percentual de cura classificado como bom por estar acima de $90 \%$; entretanto, esse coeficiente foi reclassificado como regular a partir de 2010. As regiões Norte, Nordeste e Centro-Oeste apresentaram percentual de cura na faixa regular em todos os anos de estudo.

\section{DISCUSSÃO}

Nos últimos anos, a hanseníase vem tendo decréscimo de casos em nível mundial, mas sua eliminação em alguns países ainda é desafiadora. Apesar do empenho da OMS e dos governos em erradicar a hanseníase, ela segue como problema de saúde pública (2).

No Brasil, a prevalência da hanseníase diminuiu desde o estabelecimento da segunda meta de eliminação, no ano de 2005. Entretanto, observa-se que, de 2006 a 2009, houve grande aumento na prevalência. Esse resultado pode ser explicado pelo maior empenho do Ministério da Saúde em detectar a doença, maior força das políticas de eliminação da doença e pela organização do sistema de saúde em prol da erradicação da hanseníase, com o objetivo de alcançar a meta estabelecida, até então, para

FIGURA 3. Percentual de cura da hanseníase nas regiões brasileiras, 2005, 2010 e 2015

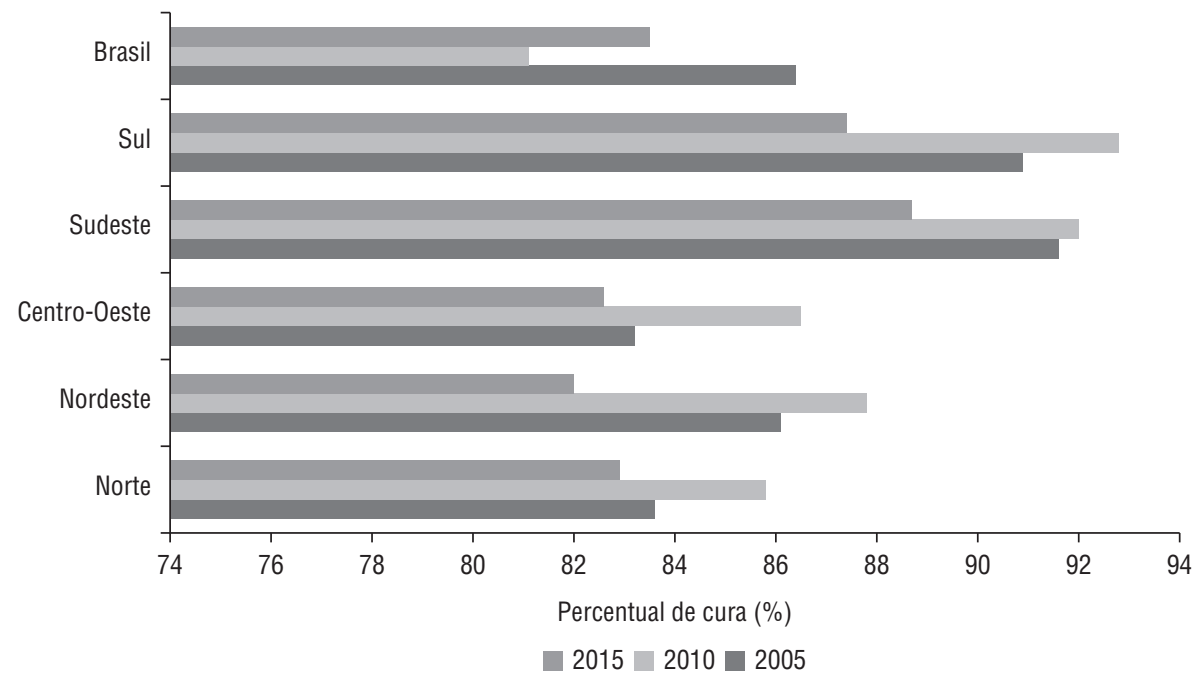

2010 - ou seja, um aumento que possivelmente não reflete um aumento real da prevalência da doença $(2,8)$.

Atualmente, o que se observa no Brasil é que, embora haja tendência de eliminação da hanseníase em nível nacional, as disparidades regionais resultam na manutenção da doença circulante. A grande extensão territorial brasileira e as desigualdades socioeconômicas entre regiões têm sido apontadas como os principais motivos dessa discrepância; de fato, as regiões mais pobres se apresentam como as mais endêmicas (8). Nesse sentido, o presente estudo corrobora pesquisas anteriores quanto à observação da irregularidade entre as regiões, que representam os extremos em termos de indicadores econômicos e de saúde (8-10): são observadas regiões com padrões de saúde comparáveis aos dos países desenvolvidos e regiões com índices de mortalidade comparáveis aos dos países mais pobres do hemisfério sul. Além disso, a disparidade entre regiões também é observada quanto aos tipos de agravos à saúde (doenças crônico-degenerativas versus doenças infecciosas e parasitárias). Tais dissemelhanças regionais são observadas, inclusive, entre diferentes grupos populacionais dentro de uma mesma região, estado ou cidade, expressando a complexidade da interação entre determinantes de saúde como desigualdades de renda, carência de alimentação, moradia, saneamento, educação e, também, dificuldade de acesso aos serviços de saúde $(8,11,12)$.

No Brasil, os maiores coeficientes de prevalência de hanseníase foram observados, em ordem decrescente, nas regiões Centro-Oeste, Norte e Nordeste. As desigualdades regionais de desenvolvimento econômico e social no Brasil têm relação histórica com a epidemiologia das doenças infectocontagiosas. As regiões Sudeste e Sul estão no extremo socioeconômico dito favorável no país. Por sua vez, Nordeste, Norte e Centro-Oeste são tradicionalmente considerados como socioeconomicamente atrasados. A baixa prevalência da doença no Sul, portanto, coincide com seu maior nível de desenvolvimento (13-16).

O acompanhamento dos indicadores em menores de 15 anos sinaliza a existência de focos ativos de transmissão e infecção recente, já que um fator de risco para infecção pelo bacilo Mycobacterium leprae é o contato com pacientes portadores da forma multibacilar, sem 
tratamento. Embora tenha havido redução da prevalência da hanseníase pela implementação da poliquimioterapia e outras medidas preventivas, ainda há elevada circulação do bacilo na sociedade. Esse aspecto pode tornar mais complexos outros desafios no controle da doença: detecção precoce, tratamento duradouro, exame de contatos e estigma $(7,17)$.

Depreende-se da análise dos dados que houve redução do coeficiente de detecção geral da hanseníase em nível nacional, apontando para uma boa expressão da política de eliminação nacional da doença. Destacam-se as regiões Nordeste e Centro-Oeste como as com maiores reduções no período estudado, apesar de serem as regiões com maior prevalência da doença. A relação que se faz é que, por serem regiões consideradas endêmicas para a hanseníase, maior destaque é dado para as ações de controle, e, por esse motivo, o sistema de saúde estaria mais sensível em detectar casos (maior prevalência) e tratá-los, justificando o menor foco ativo da doença (redução do coeficiente em menores de 15 anos) $(14,15)$.

Outro indicador padrão para a meta de eliminação da hanseníase é o coeficiente de prevalência. Em 2010, a Estratégia Global para redução da carga da hanseníase proposta pela OMS foi revista, passando a reconhecer que a redução da doença é algo complexo, que requer diminuição não apenas na prevalência, mas também no coeficiente geral de detecção e no coeficiente de detecção de casos com incapacidades grau 2 (6). A análise cruzada de tais coeficientes oferece informações sobre a eficiência operacional dos serviços envolvidos no controle da doença, visto que indivíduos diagnosticados e não curados inflam a prevalência e indicam que os serviços de saúde não estão seguindo adequadamente o protocolo terapêutico, ou ainda que há uma dificuldade de adesão dos portadores de hanseníase ao tratamento $(1,6)$.

Com a reformulação da estratégia para eliminação da hanseníase, maior destaque passou a ser dado à redução de incapacidades, o que terá impacto na redução do estigma e discriminação relacionados à doença. A redução da detecção de casos com grau 2 de incapacidade sofre influência direta da detecção precoce, pois, quanto mais cedo se diagnostica a doença, menores são as possibilidades de sequelas, da razão entre casos pauci/ multibacilares e da proporção de casos avaliados quanto ao grau de incapacidade no momento do diagnóstico. Tal coeficiente indica, então, a capacidade dos serviços de saúde de realizar o diagnóstico, tratar e curar os casos diagnosticados $(1,5,7)$.

A região Norte do Brasil apresentou aumento dos casos detectados com grau 2 de incapacidade no período, o que reflete, principalmente, o diagnóstico tardio da doença. O grau 2 de incapacidade está relacionado com a presença de sequelas e deformidades causadas pela hanseníase, indicando que o diagnóstico foi tardio ou que o seguimento clínico foi inadequado (5). Conforme comentando anteriormente, o Norte é uma das regiões que permaneceu com prevalência média no ano de 2015 (1,99/10 000 habitantes), sendo ainda uma das regiões com condições econômicas mais desfavoráveis e grande extensão territorial, com poucos estabelecimentos de saúde por habitante e pouca disponibilidade de profissionais médicos $(18,19)$.

Outro ponto importante é o grande número de casos de detecção ignorada, o que significa a existência de falhas na notificação da doença e dificulta o rastreio e o direcionamento da assistência para a doença. Esse comportamento pode estar associado às falhas na alimentação dos sistemas de informação, que é diretamente dependente do empenho dos municípios e regiões em fornecer os dados.

Apesar das diferenças regionais apontadas, a porcentagem de alta por cura tem-se mostrado similar em todo o território brasileiro. Observa-se que, de 2010 a 2015, houve redução geral na porcentagem de cura, evidenciando progressiva fragilidade do sistema (7). O indicador de cura possibilita a inferência sobre a qualidade do atendimento dos serviços de saúde à pessoa acometida pela hanseníase, expressando a efetividade dos serviços em assegurar a adesão ao tratamento até a alta. Esse dado reflete o acompanhamento íntimo proporcionado pelas equipes de saúde da família e ações ambulatoriais desenvolvidas pela atenção básica à saúde. Estima-se que a estrutura e a organização dos serviços de saúde tenham maior influência no desenho da atual situação epidemiológica da hanseníase do que os fatores socioeconômicos (12). Outras pesquisas apontam que uma maior cobertura pela Estratégia
Saúde da Família no Brasil está intimamente relacionada à redução dos focos de contágio da hanseníase e contribui para prevenir incapacidades físicas e promover adesão ao tratamento $(13,20)$.

Entre as limitações do presente estudo, é importante considerar que as informações utilizadas foram obtidas nas bases de dados oficiais do Ministério da Saúde. A disponibilização desses dados depende diretamente da atualização do sistema on-line pelas unidades de saúde municipais. Esse fator pode ter resultado em subestimação dos dados nacionais e regionais da doença. Além disso, podem ocorrer erros de notificação e diagnóstico tardio que influenciam a eliminação da doença. Outra limitação desta pesquisa foi a indisponibilidade de dados sobre hanseníase nos anos subsequentes a 2015.

Em conclusão, o estudo mostrou que os principais indicadores de hanseníase apresentaram redução no período estudado. Embora o Brasil não tenha alcançado a meta de erradicação da hanseníase, as análises apresentadas indicam que a meta estabelecida para o ano de 2020 será alcançada se for mantido o mesmo ritmo de organização estratégica e política do sistema em prol da eliminação da hanseníase.

Percebeu-se também que o ritmo lento de queda na prevalência da hanseníase pode estar relacionado a diferenças no desenvolvimento e padrão de vida entre as regiões brasileiras. Da mesma forma, a detecção precoce e a redução de incapacidades parecem estar relacionadas à eficiência dos serviços de atenção básica de saúde.

Recomenda-se que sejam realizadas novas análises com dados mais recentes que retratem a atual situação epidemiológica no Brasil. Ademais, seria prudente adaptar a política de atenção à hanseníase à realidade de cada região brasileira, visto que, devido à grande extensão territorial e distribuição irregular de recursos no país, a prevalência da doença é heterogênea.

Conflitos de interesse. Nada declarado pelos autores.

Declaração. As opiniões expressas no manuscrito são de responsabilidade exclusiva dos autores e não refletem necessariamente a opinião ou política da RPSP/PAJPH ou da Organização PanAmericana da Saúde (OPAS). 


\section{REFERÊNCIAS}

1. Brasil, Ministério da Saúde, Secretaria de Vigilância em Saúde. Boletim Epidemiológico. Situação epidemiológica da hanseníase no Brasil - análise de indicadores selecionados na última década e desafios para eliminação. Bol Epidemiol. 2013;44(11):1-12.

2. Brasil, Ministério da Saúde, Secretaria de Vigilância em Saúde, Departamento de Vigilância em Doenças Transmissíveis. Plano integrado de ações estratégicas de eliminação da hanseníase, filariose, esquistossomose e oncocercose como problema de saúde pública, tracoma como causa de cegueira e controle de geohelmintíases: plano de ação 2011-2015. Brasília: Ministério da Saúde; 2012.

3. Brasil, Ministério da Saúde, Departamento de Atenção Básica. Legislação sobre o controle da hanseníase no Brasil. Brasília: Ministério da Saúde; 2000.

4. World Health Organization (WHO). Leprosy global situation. Wkly Epidemiol Rec. 2005;34:289-96.

5. Brasil, Ministério da Saúde. Plano Nacional de Eliminação da Hanseníase em nível municipal 2006-2010. Brasília: Ministério da Saúde; 2006.

6. Organização Mundial da Saúde (OMS). Estratégia global aprimorada para redução adicional da carga da hanseníase: 2011-2015: diretrizes operacionais (atualizadas). Brasília: Organização PanAmericana da Saúde; 2010.

7. Brasil, Ministério da Saúde, Secretaria de Políticas de Saúde, Departamento de Atenção Básica. Guia para o controle da hanseníase. Brasília: Ministério da Saúde; 2002.

8. Brasil, Ministério da Saúde, Secretaria de Vigilância em Saúde, Departamento de Vigilância das Doenças Transmissíveis. Exercício de monitoramento da eliminação da hanseníase no Brasil - LEM-2012. Brasília: Ministério da Saúde; 2015.

9. Vasconcelos AMN, Gomes MMF. Demographic transition: the Brazilian experience. Epidemiol Serv Saude. 2012; 21(4):539-48.

10. Alves DB, Barbosa MTS. Desigualdades na mortalidade por doenças crônicas entre idosos e sua associação com indicadores socioeconômicos no Brasil. Rev Bras Cienc Envelh Hum. 2010;7(1):22-33.

11. Araujo JD. Polarização epidemiológica no Brasil. Epidemiol Serv Saude. 2012; 21(4):533-8.

12. Barros MBA, César CLG, Carandina L, Torre GD. Desigualdades sociais na prevalência de doenças crônicas no Brasil, PNAD-2003. Cienc Saude Coletiva. 2006; 11(4):911-26.

13. Magalhaes MCC, Rojas LI. Diferenciação territorial da hanseníase no Brasil. Epidemiol Serv Saude. 2007;16(2):75-84.

14. Sanches LAT, Pittner E, Sanches HF, Monteiro MC. Detecção de casos novos de hanseníase no município de Prudentópolis, PR: uma análise de 1998 a 2005. Rev Soc Bras Med Trop. 2007;40(5):541-5.

15. Amaral EP, Lana FCF. Análise espacial da Hanseníase na microrregião de Almenara, MG, Brasil. Rev Bras Enferm. 2008;61(spe): 701-7.
16. Andrade MV, Noronha KVMS, Menezes RM, Souza MN, Reis CB, Martins DR, et al. Desigualdade socioeconômica no acesso aos serviços de saúde no Brasil: um estudo comparativo entre as regiões brasileiras em 1998 e 2008. Econ Apl. 2013;17(4): 623-45.

17. Lana FCF, Amaral EP, Lanza FM, Lima PL de Carvalho ACN, Diniz LG. Hanseníase em menores de 15 anos no Vale do Jequitinhonha, Minas Gerais, Brasil. Rev Bras Enferm. 2007;60(6):696-700.

18. Instituto de Pesquisa Econômica Aplicada (IPEA). Presença do Estado no Brasil: federação, suas unidades e municipalidades Brasília: IPEA; 2009. Disponível em: http://www.ipea.gov.br/presenca / index.php?option=com_content\&view= article\&id=13\&Itemid=12 Acessado em 14 de julho de 2017.

19. Silva RGS, Vidal MB. Níveis de eficiência nos serviços de saúde pública na região norte. Rev Desenvolv Econ. 2008;18: 156-65.

20. Lanza FM. Avaliação da atenção primária no controle da hanseníase: validação de instrumentos e análise do desempenho de municípios endêmicos do Estado de Minas Gerais [tese]. Belo Horizonte: Escola de Enfermagem da UFMG; 2014.

Manuscrito recebido em 13 de abril de 2017. Aceito em versão revisada em 27 de julho de 2017.
ABSTRACT

Epidemiologic study of leprosy in Brazil: reflections on elimination goals
Objective. To describe the epidemiological profile of leprosy in Brazil in the period from 2005 to 2015 and describe the behavior of leprosy indicators in relation to the goals established by the World Health Organization (WHO) for elimination of this disease.

Method. The study was performed using data from the Ministry of Health websites. The following indicators were assessed: prevalence, overall coefficient of detection, coefficient of detection according to age ( $<15$ years or $\geq 15$ years), cure rate, and proportion of cases with WHO grade 2 disability.

Results. During the study period, the prevalence of leprosy cases was stable at a medium level (1.00 to 4.99/10 000 population), with decreasing national trend. However, the national trend was not observed in the Northeast, North, and Midwest regions. The coefficient of new cases in individuals $<15$ years of age decreased from 2005 to 2015 in all regions. A marked decrease was detected in the proportion of new cases with grade 2 disability, especially in the South and Southeast. The North was the only region with increase in this indicator. The rate of leprosy cure in Brazil has not changed since 2005, having remained within the $75-90 \%$ range regardless of age group. Conclusion. There was improvement (reduction) in the main leprosy indicators in Brazil from 2005 to 2015. Even though Brazil did not eradicate leprosy, this goal will likely be reached in 2020 if the current parameters are maintained. Leprosy control policies should be adapted to the reality of each Brazilian region, given the heterogeneous distribution of prevalence.

Keywords Epidemiology; leprosy; disease eradication; Brazil. 
RESUMEN Objetivos. Describir el perfil epidemiológico de la lepra en el Brasil en el período 2005-2015 y verificar cómo se comportan los indicadores brasileños en relación con

Estudio epidemiológico de la lepra en el Brasil: reflexión sobre las metas de eliminación las metas establecidas por la Organización Mundial de la Salud (OMS) para la eliminación de esa enfermedad.

Métodos. La investigación se realizó a partir de los datos disponibles en los sitios del Ministerio de Salud. Los indicadores evaluados fueron los datos de prevalencia, los coeficientes de detección general y por grupo etario ( $<15 \mathrm{o} \geq 15$ años), el porcentaje de curación y la discapacidad de grado 2.

Resultados. En el período del estudio, el coeficiente de prevalencia de casos de lepra se mantuvo en un nivel promedio (de 1,00 a 4,99/10 000 habitantes), con una tendencia nacional decreciente. Sin embargo, en las regiones del nordeste, norte y centro-oeste no se observó ese comportamiento. El coeficiente de casos nuevos en menores de 15 años disminuyó entre el 2005 y el 2015 en todas las regiones. El coeficiente de detección de casos nuevos con diagnóstico de discapacidad de grado 2 tuvo una reducción significativa, principalmente en las regiones del sur y del sudeste; el norte del país fue la única región donde aumentó ese coeficiente. El porcentaje de curación de la lepra en el Brasil no ha cambiado desde el 2005 y se considera regular (de 75\% a 90\%), independientemente del grupo de edad.

Conclusión. Los principales indicadores de lepra se redujeron en el período del estudio. Aunque no se haya erradicado la lepra en el Brasil, esa meta deberá alcanzarse en el 2020 si se mantienen los parámetros. Se recomienda adaptar la política de atención a la lepra a la realidad de cada región brasileña, en vista de que la prevalencia de la enfermedad presenta una distribución heterogénea.

Palabras clave Epidemiología; lepra; erradicación de la enfermedad; Brasil. 